\title{
Evaluation of Podocalyxin level in pre-eclampsia with severe features' patients: a cross-sectional study
}

\author{
Ahmed F. Amin', Hisham Abou-Taleb', Mustafa Gamal², Marwa M. Thabet ${ }^{3}$, Nashwa Azoz ${ }^{4}$, \\ Ahmed M. Abbas ${ }^{1 *}$
}

\author{
${ }^{1}$ Department of Obstetrics and Gynecology, Faculty of Medicine, Assiut University, Assiut, Egypt \\ ${ }^{2}$ Department of Obstetrics and Gynecology, Luxor International Hospital, Luxor, Egypt \\ ${ }^{3}$ Department of Clinical Pathology, Assiut University, Assiut, Egypt \\ ${ }^{4}$ Department of Internal Medicine Faculty of Medicine, Assiut University, Assiut, Egypt
}

Received: 06 June 2019

Accepted: 03 July 2019

\section{*Correspondence:}

Dr. Ahmed M. Abbas,

E-mail: bmr90@hotmail.com

Copyright: () the author(s), publisher and licensee Medip Academy. This is an open-access article distributed under the terms of the Creative Commons Attribution Non-Commercial License, which permits unrestricted non-commercial use, distribution, and reproduction in any medium, provided the original work is properly cited.

\section{ABSTRACT}

Background: This study aims to evaluate the level of podocalyxin (PCX) in preeclampsia with severe features patients and correlate it with the results of laboratory tests.

Methods: The current study was a cross-sectional study conducted in Assiut Women Health Hospital between April and October 2018. The study included 60 patients divided into two groups; Group (A): 30 patients diagnosed to have preeclampsia with severe features and Group (B): 30 patients as normal control group. Complete laboratory investigations with measurements of the PCX level was performed for all study participants.

Results: No statistically significant difference between the study group and control group according to blood urea ( $\mathrm{p}=$ $0.339)$ and serum creatinine $(\mathrm{p}=0.801)$. There was statistically significant difference between the study group and control group according to PCX level $(\mathrm{p}=0.001)$; the mean PCX was $3340.0 \pm 2394.6$ in the study group versus $1083.5 \pm 1400.2$ in the control group. Univariate analysis revealed podocalyxin was not correlated with clinical data or laboratory investigations.

Conclusions: Podocalyxin levels were significantly elevated in preeclampsia.

Keywords: High risk pregnancy, Hypertension with pregnancy, Podocalyxin, Preeclampsia, Renal function tests, Renal injury

\section{INTRODUCTION}

Hypertensive disorders of pregnancy could be classified into 4 categories: gestational hypertension, preeclampsiaeclampsia, chronic hypertension, and preeclampsia superimposed upon underlying hypertension. Diagnosis generally depends on gestational age at the time of presentation. Distinguishing preeclampsia from other causes of hypertension can be difficult but is essential to maternal and fetal outcomes. ${ }^{1}$ Pre-eclampsia is a leading cause of maternal mortality, responsible annually for over 60,000 maternal deaths around the world. Pre-eclampsia is a multisystem disease featuring hypertension, proteinuria, and renal, hepatic, and neurological involvement. Diagnosis is often elusive, as clinical presentation is highly variable. ${ }^{2}$

The characteristic kidney lesion of pre-eclampsia is swelling and detachment of glomerular endothelial cells. This is often associated with subendothelial deposits, 
causing capillary lumina obstruction. Pre-eclampsia is associated with altered hemodynamic abnormalities such as lowered renal plasma flow, reduction in GFR by $30 \%-$ $40 \%$, and renal vasoconstriction. Thus, kidney in preeclamptic women is highly susceptible to ischemic injury. Renal tubular secretion of uric acid is impaired in pre-eclampsia, resulting in elevated concentration of uric acid in the blood. The urine sediment is usually bland. Acute kidney injury (AKI) occurs in about $1 \%$ of women with severe pre-eclampsia. ${ }^{3}$

Podocytes are highly specialized glomerular visceral epithelial cells. Their function is the stabilization of the glomerular capillaries and participation in the barrier function of the glomerular filter. A growing body of evidence suggests that podocyte damage takes place in preeclampsia, and is reflected by the abnormal shedding of podocytes in the urine. Several markers of podocyte injury are available (i.e. nephrin, sinaptopodin, podocalyxin, podocin). Some of them are more specific to the podocytes, and some are also expressed in other tissues. $^{4}$

Podocalyxin (PCX), a sialomucin most closely related to CD34 and endoglycan, is expressed by podocytes, hematopoietic progenitors, vascular endothelia, and a subset of neurons. The PCX function is to maintain podocyte shape and distortion of the slit diaphragm. PCX is usually located on the apical cell membrane of podocytes and is shed into urine from injured podocytes. ${ }^{5}$

This study aims to evaluate the level of PCX in preeclampsia with severe features patients and correlate it with the results of laboratory tests.

\section{METHODS}

The current study was a cross-sectional study conducted in Assiut Women Health Hospital between April and October 2018. The Institutional Ethical Review board approved the study. All patients signed informed written consent before participation in the study.

Patients were divided into two groups; Group (A): 30 patients diagnosed to have preeclampsia with severe features and Group (B): 30 patients as normal control group.

\section{Inclusion criteria}

- Age: 18-35 years

- Singleton pregnancy

- Gestational age 20 weeks or older

- Severe Features of Preeclampsia.

\section{Exclusion criteria}

- Patients with prior history of chronic kidney disease, glomerulonephritis, hematuria, autoimmune disorders
- Chronic corticosteroid drug use

- Oliguria (<400 mL of urine output in 24 hours)

- Intrauterine fetal death

- Nephrotoxic drugs use.

The women were subjected to the following:

1. Detailed history taking

2. Clinical examination:

a) General examination: pulse, temperature, body weight and height, body mass index. Blood pressure was measured in ER in semi setting, using adequate sized cuff and on two occasions at least 4 hours apart.

b) Abdominal examination.

3. Obstetric Ultrasonography : to calculate gestational age, viability, amniotic fluid, placental site and to exclude any congenital malformation

4. Assessment of renal function: urine output, blood urea, serum creatinine, serum uric acid, eGFR

-eGFR was calculated by CKD-EPI equation by serum creatinine level (according to KDIGO 2012).

5. C.B.C., Rh, blood grouping, liver function tests.

\section{Sample collection}

Urine analysis was done in ER by Urine Dipstick Analysis (uri-trak-3). (10 ml) of midstream urine was collected in clean plastic tubes, without preservative. all samples were centrifuged for clarification (at 3,000 rpm for $5 \mathrm{~min}$ ), then stored at $-80^{\circ} \mathrm{C}$ and collectively analyzed once at the clinical pathology laboratory at the women Health University Hospital.

- Prior to the assay, the samples were allowed to thaw at room temperature $\left(24^{\circ} \mathrm{C}\right)$. All assays were completed using duplicate wells for each dilution of the standard and each dilution of the sample.

- A commercially available Podocalyxin ELISA test (Exocell Inc.) was used for evaluation of the tested biomarker. All assays were performed following the manufacturer's instructions including all standard cure range, sample dilution.

- ALL staff who performed the assays of analytes were blinded to the clinical information.

Patients were managed according to RCOG guidelines which include termination of pregnancy, antihypertensive drugs and sedatives

\section{Statistical analysis}

Statistical analysis was performed using SPSS software version 20 (SPSS, Chicago, IL, USA). For Independent samples: Student's t-test was used for comparing normal distribution numerical data between two groups, and Chi square or Fisher's exact tests for categorical data. 
Correlation between the level of PCX and laboratory markers was done by spearman's correlation test. $\mathrm{p}<0.05$ is considered to be significant.

\section{RESULTS}

There was no statistically significant difference between the study group and control group according to age $(\mathrm{p}=$ 0.677 ); the mean age was $22.93 \pm 4.77$ in the study group and $23.47 \pm 5.00$ in the control group. The mean BMI was $22.47 \pm 3.81$ in the study group and $22.17 \pm 3.62$ in the control group $(\mathrm{p}=0.743)$.

Similarly, no statistically significant difference between the study group and control group regarding to gestational age $(\mathrm{p}=0.169)$; the mean gestational age was $35.20 \pm 2.19$ in the study group and $36.07 \pm 2.49$ in the control group. There was no statistically significant difference between both groups regarding to parity $(\mathrm{p}=$ 0.853).

There was no statistically significant difference between the study group and control group according to blood urea $(p=0.339)$; the mean blood urea was $31.98 \pm 13.64$ in the study group and $29.30 \pm 12.95$ in the control group. Also, no statistically significant difference between the study group and control group according to serum creatinine $(p=0.801)$; the mean serum creatinine was $0.78 \pm 0.27$ in the study group and $0.79 \pm 0.26$ in the control group.

Table 1: Comparison between study group and control group as regard podocalyxin.

\begin{tabular}{|c|c|c|c|}
\hline Podocalyxin & $\begin{array}{l}\text { Study } \\
(\mathrm{n}=\mathbf{3 0})\end{array}$ & $\begin{array}{l}\text { Control } \\
(\mathrm{n}=30)\end{array}$ & $\begin{array}{l}P \text { - } \\
\text { value }\end{array}$ \\
\hline Mean \pm SD & $3340.0 \pm 2394.6$ & $1083.5 \pm 1400.2$ & \multirow{2}{*}{$\begin{array}{l}0.001 \\
*\end{array}$} \\
\hline $\begin{array}{l}\text { Median } \\
\text { (Range) }\end{array}$ & $\begin{array}{l}4169.5(246- \\
7165)\end{array}$ & $\begin{array}{l}464.5(271.0- \\
4957.5)\end{array}$ & \\
\hline
\end{tabular}

* Statistically significant difference

Table 2: Correlation of podocalyxin with clinical data and laboratory investigations.

\begin{tabular}{|lll|}
\hline Variables & $\begin{array}{l}\text { Podocalyxin } \\
\text { r-value }\end{array}$ & P-value \\
\hline Systolic B.P & 0.259 & 0.211 \\
\hline Diastolic B.P & 0.004 & 0.986 \\
\hline Blood urea & -0.091 & 0.666 \\
\hline Serum creatinine & -0.242 & 0.244 \\
\hline HB & 0.103 & 0.625 \\
\hline Platelet count & 0.072 & 0.733 \\
\hline
\end{tabular}

BP; blood pressure, HB; hemoglobin

There was statistically significant difference between the study group and control group according to PCX level $(p=0.001)$; the mean PCX was $3340.0 \pm 2394.6$ in the study group versus $1083.5 \pm 1400.2$ in the control group (Table 1). Univariate analysis revealed podocalyxin was not correlated with clinical data or laboratory investigations (Table 2).

\section{DISCUSSION}

Podocalysin is the major sialoglycoprotein present on the surface of podocytes. The amount of its urinary excretion is associated with the degree of podocyte damage in glomerular diseases. ${ }^{4}$ Unlike other hypertensive pregnancy disorders, pre-eclampsia is a systemic disease with multi-organ involvement, which is commonly, but not always, associated with by either sudden onset or worsening of pre-existing proteinuria. ${ }^{6}$

In the present study, we found statistically significant difference between the study group and control group according to PCX level $(\mathrm{p}=0.001)$; the mean PCX level was $3340.0 \pm 2394.6$ in the study group and $1083.5 \pm 1400.2$ in the control group.

Chen et al, found that PCX serum levels were significantly elevated in preeclampsia, especially the early-onset subtype. Within the placenta, blood vessels but not trophoblasts expressed podocalyxin, and preeclampsia didn't differ from controls. ${ }^{7}$

Wang et al, found that ,in preeclampsia, urinary nephrin and PCX concentrations were significantly increased and highly correlated to each other, $r(2)=0.595$. Nephrin and PCX were also correlated with urine protein concentrations. $^{8}$

In Palacios de Franco et al, study, podocyturia (expressed as urinary podocalyxin) was shown to be elevated in cases of preeclampsia and eclampsia (4). This association remained independent of proteinuria and correlated with UPC. Also, its values appeared to normalize after delivery (to levels comparable to those seen in normal pregnancies), in the same manner as the proteinuria and blood pressure readings. This finding is somewhat similar to the only other study of urine podocalyxin (detected by ELISA) in preeclampsia published so far, by Wang et al. Nonetheless, it is noted that in that study, patients with chronic hypertension also had a tendency to higher levels of urine podocalyxin. ${ }^{8}$

Our results showed statistically significant difference between the study group and control group regarding GFR. Krutzén et al, found that in subjects with preeclampsia the rise in GFR observed in normal pregnancy was absent, the development of proteinuria and fluid retention typical of preeclampsia is paralleled by a deterioration of GFR. ${ }^{9}$

There was none significant relation between GFR and podocalyxin. Podocalyxin was not correlated with laboratory investigations. In Hara et al, urinary podocalyxin levels were not correlated with other laboratory markers such as blood pressure, lipid level, serum creatinine, estimated GFR or proteinuria. ${ }^{10}$ 
In women who go on to develop preeclampsia, uric acid concentration is elevated as early as 10 weeks of gestation, a time much earlier than the clinical presentation of the disorder. Increased uric acid often precedes clinical manifestations of the disease, involving reduced GFR. ${ }^{11}$ Vyakaranm et al, recorded an increase in the mean uric acid and creatinine values among preeclamptic patients. ${ }^{12}$

\section{CONCLUSION}

Podocalyxin levels were significantly elevated in preeclampsia Biomarkers of glomerular injury such as podocalyxin show promise as diagnostic aids in preeclampsia.

\section{Recommendations}

The current study has certain limitations. Due to the relatively small sample size, future studies with a larger sample size are needed to further investigate the association between serum podocalyxin and preeclampsia. In addition, it is unknown whether podocalyxin is elevated in preeclampsia cases without proteinuria; also the levels of podocalyxin at which the pregnancy have to be terminated are needed to be investigated in the future.

Funding: No funding sources Conflict of interest: None declared

Ethical approval: The study was approved by the Institutional Ethics Committee

\section{REFERENCES}

1. Lai C, Coulter SA, Woodruff A. Hypertension and Pregnancy. Tex Heart Inst J. 2017;44(5):350-1.

2. Duhig KE, Shennan AH. Recent advances in the diagnosis and management of pre-eclampsia. F1000 Prime Rep. 2015;7:24.

3. Prakash J, Ganiger VC. Acute Kidney Injury in Pregnancy-specific Disorders. Indian J Nephrol. 2017;27(4):258-70.

4. Palacios de Franco Y, Velazquez K, Segovia N. Urinary podocalyxin as a marker of preeclampsia in a Hispanic population. Int $\mathbf{J}$ Physiol Pathophysiol Pharmacol. 2014;6(2):115-24.
5. Asao R, Asanuma K, Kodama F. Relationships between levels of urinary podocalyxin, number of urinary podocytes, and histologic injury in adult patients with IgA nephropathy. Clin J Am Soc Nephrol. 2012;7(9):1385-93.

6. Craici IM, Wagner SJ, Weissgerber TL, Grande JP, Garovic VD. Advances in the pathophysiology of pre-eclampsia and related podocyte injury. Kidney Int. 2014;86(2):275-85

7. Chen Q, Wang Y, Li Y, Zhao M, Nie G. Serum podocalyxin is significantly increased in early-onset preeclampsia and may represent a novel marker of maternal endothelial cell dysfunction. J Hypertens. 2017;35(11):2287-94.

8. Wang Y, Zhao S, Loyd S, Groome LJ. Increased urinary excretion of nephrin, podocalyxin, and Bigh3 in women with preeclampsia. Am J Physiol Renal Physiol. 2012;302(9):F1084-9.

9. Krutzén E, Olofsson P, Bäck SE, Nilsson-Ehle P. Glomerular filtration rate in pregnancy: a study in normal subjects and in patients with hypertension, preeclampsia and diabetes. Scand J Clin Lab Invest. 1992;52(5):387-92.

10. Hara M, Yamagata K, Tomino Y. Urinary podocalyxin is an early marker for podocyte injury in patients with diabetes: establishment of a highly sensitive ELISA to detect urinary podocalyxin. Diabetologia. 2012;55(11):2913-9.

11. Roberts JM, Bodnar LM, Lain KY, Hubel CA, Markovic N, Ness RB, Powers RW. Uric acid is as important as proteinuria in identifying fetal risk in women with gestational hypertension. Hypertension. 2005;46:1263-9.

12. Vyakaranam S, Bhongir A, Patlolla D, Chintapally R. Study of serum uric acid and creatinine in hypertensive disorders of pregnancy. International Journal of Medical Science and Public Health. 2015;4(10):1424-8.

Cite this article as: Amin AF, Abou-Taleb H, Gamal M, Thabet MM, Azoz N, Abbas AM. Evaluation of Podocalyxin level in pre-eclampsia with severe features' patients: a cross-sectional study. Int J Reprod Contracept Obstet Gynecol 2019;8:3255-8. 